\title{
Quantification of Fungal Antigens in Soil with a Monoclonal Antibody-Based ELISA: Analysis and Reduction of Soil-Specific Bias
}

\author{
W. Otten, C. A. Gilligan, and C. R. Thornton
}

Department of Plant Sciences, University of Cambridge, Downing Street, Cambridge CB2 3EA, United Kingdom. Accepted for publication 17 April 1997.

\begin{abstract}
Otten, W., Gilligan, C. A., and Thornton, C. R. 1997. Quantification of fungal antigens in soil with a monoclonal antibody-based ELISA: Analysis and reduction of soil-specific bias. Phytopathology 87:730-736.

This paper describes methods to improve the use of immunoassays for quantification of soilborne fungal antigens. Calibration curves, prepared by diluting known quantities of an antigen into soil extracts and into soil, were described by a four-parameter logistic curve from which two principal criteria, the lower detection limit and the horizontal locational parameter, were used to summarize the sensitivity and bias of an immunoassay. We identify two sources of bias, retention of the antigen in soil due to bonding and interference of soluble soil components in plate-trappedantigen, enzyme-linked immunosorbent assays. Using a monoclonal
\end{abstract}

ABSTRACT antibody that recognizes a putative catechol oxidase secreted by hyphae of Rhizoctonia solani, we show that bias due to retention of the antigen in soil is substantially greater than bias due to interference. Three soils were compared: a sand, a clay, and a loam. The degree of retention varied with soil type, with more than a 1,000-fold reduction in sensitivity in the clay soil. Addition of $\mathrm{CuSO}_{4}$ to the extraction solution and optimizing the volume of extractant reduced the bias and increased the sensitivity of the assay for all three soils. Possible mechanisms for the effect due to $\mathrm{Cu}^{2+}$ and the implications for the design and use of calibration curves for assays involving quantification of fungal antigens in soil are discussed.

Additional keywords: antigen recovery, freeze-dried mycelium.
Monoclonal antibody assays hold considerable potential for the detection and quantification of economically important, soilborne fungal pathogens (5), but, with the exception of a few cases (15, 18-20), this potential has not yet been fully realized. One of the key factors determining the exploitation of soil-based immunoassays is the ability to recover significant amounts of the antigen when it is present in soil. Other factors include the dynamics of antigen production by the target fungus, the degradation of the antigen in soil, and the relationship between antigen secretion and fungal biomass. Each of these factors requires careful analysis and testing in the development of a robust assay for the quantification of soilborne fungi (6). It follows, however, that even when the dynamics of antigen production by the fungus and of breakdown of the antigen in the soil are known, the sensitivity of a potentially good assay may be lost by the retention of the antigen in soil.

Retention of the antigen may occur on components of the soil solid phase by a range of physicochemical processes including electrostatic bonding by ion exchange after protonation and by hydrogen bonding, in particular between $\mathrm{N}-\mathrm{H}$ groups and organic compounds. This is especially important in soils high in organic matter, since humic soil materials are known to interact with a variety of organic compounds such as proteins, amino acids, and carbohydrates (17), which commonly form the building blocks of fungal antigens (6). The amount and purity of the antigen recovered from soil is, therefore, likely to depend intimately on soil physical and chemical properties. An additional form of soil-specific bias may occur in the microtiter wells of a plate-trapped assay, when soluble components extracted from a soil affect the immobilization of fungal antigens onto the well surface. Unlike interference

Corresponding author: W. Otten; wo200@cus.cam.ac.uk

Publication no. P-1997-0602-01R

(C) 1997 The American Phytopathological Society in enzyme-linked immunoassays by plant extracts, interference by soil components has received little attention $(1,9,22)$.

In this paper, we introduce known quantities of an antigen extracted from freeze-dried mycelium (FDM) of the ubiquitous plant pathogenic fungus Rhizoctonia solani Kühn into soil in order to analyze the effect of retention of the antigen within soil. The antigen is a putative catechol oxidase that is representative of a broad class of glycoprotein antigens (C. R. Thornton, unpublished data). Specifically, we (i) quantify the magnitude of the bias in estimation of the antigen due to incomplete extraction from soil; (ii) quantify the bias in estimation of the antigen due to interference by soluble soil components in the assay; (iii) test whether or not bias due to retention of the antigen and interference differ amongst three soils; (iv) show how recovery of the antigen can be improved by inclusion of metal cations in the extraction buffer and by optimizing the volume of extractant; and (v) present a protocol to compensate for incomplete extraction in soils and interference by soil components for a plate-trapped immunoassay. Particular attention is given to the nonlinear relationship between antigen and absorbance in order to allow for unequal effects in retention over a range from low to high antigen concentrations. The broader implications of the analyses and assay protocols for the quantification of antigens secreted by soilborne fungi are discussed.

\section{MATERIALS AND METHODS}

Solutions containing soluble fungal antigens were analyzed by a plate-trapped-antigen, enzyme-linked immunosorbent assay (PTAELISA). Bias in the assay due to soluble components extracted from soil and retention of the antigen by solid soil components was quantified by comparison with reference samples over the entire workable absorbance range. We refer to bias due to soluble components as interference, and bias due to incomplete extraction from soil as retention. The immunoglobulin M (IgM) monoclonal antibody (MAb) used in this study, designated EE1, was devel- 
oped and described by Thornton et al. $(18,19)$. The MAb recognizes a carbohydrate epitope that forms part of a larger glycoprotein molecule, the enzyme catechol oxidase (EC 1.10.3.1).

Reference calibration curve. In the absence of a source of purified antigen, we used antigen extracted from a known amount of FDM as a standard. FDM of an anastomosis group 4 isolate of Rhizoctonia solani (R3) was prepared according to the procedure outlined by Thornton et al. (18). Antigen was extracted from FDM in phosphate-buffered saline (PBS; $0.8 \% \mathrm{NaCl}, 0.02 \% \mathrm{KCl}, 0.115 \%$ $\mathrm{Na}_{2} \mathrm{HPO}_{4}$, and $0.025 \% \mathrm{KH}_{2} \mathrm{PO}_{4} ; \mathrm{pH} 7.2$ ), contained in $1.5-\mathrm{ml} \mathrm{mi}-$ crocentrifuge tubes, using a hand-held homogenizer. Fungal mycelium was pelleted by centrifugation at $12,000 \times g$ for $10 \mathrm{~min}$ and supernatant containing solubilized antigen transferred to 12-well microtiter strips (catalog no. 9502107; Labsystems Oy, Helsinki, Finland) for preparation of reference calibration curves. Curves were constructed by serial dilution of the antigen extract into fresh PBS. There were 12 twofold dilutions per curve. Wells containing antigen in a 50- $\mu$ l volume were incubated overnight $(16 \mathrm{~h})$ at $4^{\circ} \mathrm{C}$, and then given three 3-min washes with PBST (PBS + 0.05\% [vol/ vol] Tween 20 [polyoxyethylene-sorbitan monolaurate]; P-1379; Sigma Chemical Co., Poole, United Kingdom) and a single 1-min wash with PBS followed by distilled water. Wells were air-dried for $15 \mathrm{~min}$ in a laminar flow cabinet and stored at $4^{\circ} \mathrm{C}$ in sealed plastic bags until assayed by PTA-ELISA, as described below. The effect of variability in the relationship between antigen and absorbance was quantified by ELISA with curves assayed on the same day but on different plates, and with curves assayed on different days. Antigen for these calibrations was obtained from the same stock source. Twenty calibration curves were divided over five plates and analyzed on the same day to quantify the effect of variability among plates. In separate experiments, variability among days in the ELISA was quantified for curves prepared from the same stock source of FDM, but analyzed on separate days.

Bias due to interference. To quantify the effect of soluble soil components on the detection of the EE1 antigen, separate calibration curves were prepared by diluting a solution containing the antigen extracted from a known amount of FDM in extracts from three different soils, together with a control prepared and diluted in PBS alone. The bias due to interference was computed by the difference between the control and test curves in the soil extracts. The test soils comprised a sand (acid-washed sand, grade 16/30, pH 7.0; Hepworth Minerals and Chemicals Limited, Redhill, United Kingdom), a clay soil (sandy clay loam, clay content $23.8 \%$; organic matter content $2.1 \%$; and cation exchange capacity of $16.4 \mathrm{meq} /$ $100 \mathrm{~g} ; \mathrm{pH}-\mathrm{H}_{2} \mathrm{O}$ 7.6; Cambridge University Field Station, Cambridge), and a loam soil (loamy sand, clay content $6.85 \%$; organic matter content $1.1 \%$; and cation exchange capacity of $7.7 \mathrm{meq} / 100$ $\mathrm{g} ; \mathrm{pH}-\mathrm{H}_{2} \mathrm{O}$ 6.7; from Woburn, United Kingdom). The clay and loam were air-dried and sieved to a fraction of 500 to $1,000 \mu \mathrm{m}$ prior to use. All soils were initially tested by ELISA for absence of Rhizoctonia. To obtain extracts from each soil, PBS was added at a ratio of $1.5 \mathrm{ml}$ of buffer to $1 \mathrm{~g}$ of solid. The PBS solution was mixed for $4 \mathrm{~h}$ at room temperature $\left(23 \pm 2^{\circ} \mathrm{C}\right)$, followed by centrifugation at $12,000 \times g$ for $10 \mathrm{~min}$. Dilution series, using a solution containing the antigen extracted from FDM prepared and subsequently diluted in these soil extracts, were constructed in microtiter wells and analyzed by PTA-ELISA, as described below. There were 12 twofold dilutions per curve, with three replicates per dilution.

Bias due to retention. Retention of the antigen in soil was quantified by mixing soil with solutions containing solubilized antigen extracted from known amounts of FDM. The resulting calibration curves include the combined effects of interference and retention, since, as a result of mixing the solution with soil, other components were extracted from the soil that may interfere in the assay. The effect due to retention was computed by the horizontal shift between these curves and the calibration curves prepared with soil extract. Antigen dilution series were prepared in $25-\mathrm{ml}$ polyvials containing fresh PBS. The undiluted extract contained solubilized antigen from $30 \mathrm{mg}$ of FDM per milliliter of PBS. There were 12 twofold dilutions for each of the test soils, with three replicates per dilution. One-gram dry weight samples of sieved soil were added to $1.5 \mathrm{ml}$ of extract from each of the dilutions. Samples were shaken in the dark for $4 \mathrm{~h}$ at room temperature and then centrifuged at $12,000 \times g$ for $10 \mathrm{~min}$. Supernatants were transferred to microtiter wells and analyzed by PTA-ELISA, as described below.

Reduction of bias. Preliminary work (data not shown) to compare the efficacy of different extraction buffers, including the effects of $\mathrm{pH}$, magnesium, copper, and salts on extraction of the EE1 antigen, showed that $\mathrm{CuSO}_{4}$ was most successful in increasing extraction of the antigen from soils. Accordingly, calibration curves in the extractant alone, in soil extract, and in soil were prepared using a $\mathrm{CuSO}_{4}$ solution $\left(0.1 \mathrm{M} \mathrm{CuSO}_{4}, 0.8 \% \mathrm{NaCl}\right.$, and $0.02 \% \mathrm{KCl} ; \mathrm{pH} 4.2$ ). The three curves were used to quantify retention (soil extract versus soil) and interference (extractant versus soil extract). Samples were prepared as described previously and analyzed by PTA-ELISA.

The optimal buffer:soil ratio for antigen extraction and detection was determined for samples obtained from samples that contained live, actively growing mycelium. We looked for a trend or optimum in the response of absorbance to buffer:soil ratio. A source of nutrient-enriched inoculum, hereafter referred to as chopped potato soil inoculum (CPS), was prepared using clay soil according to the method described in Thornton et al. (18). The CPS was airdried and sieved to a fraction of 500 to $1,000 \mu \mathrm{m}$. Sand and a claysand mixture (1:1 wt/wt sand to clay) contained in separate polystyrene boxes $(12 \times 8 \times 2 \mathrm{~cm})$ were mixed thoroughly with $1 \%$ (in the sand) or $10 \%$ (in the clay-sand) (wt/wt) CPS and wetted to $12 \%$ (vol/wt) moisture with distilled water. These microcosms comprising infested clay-sand mixture or sand were incubated at $23^{\circ} \mathrm{C}$ for 2 days (sand) or 3 days (clay-sand) prior to sampling. Antigens were extracted from the microcosms by adding 0.5 to $5.0 \mathrm{ml}$ of extractant solution (PBS or $\mathrm{CuSO}_{4}$ ) in $0.5-\mathrm{ml}$ stepwise increments of volume to triplicate 1-g weight samples, followed by shaking for 4 $\mathrm{h}$ at room temperature. Extracts were centrifuged, immobilized in microtiter wells, and analyzed by PTA-ELISA, as described below.

Quantification of sensitivity and bias. The characteristic sigmoidal calibration curve between absorbance $(A)$ and the logarithm of the concentration of antigen extracted from FDM (expressed as milligrams per milliliter) is summarized, for convenience, by a logistic function with four parameters

$$
A=\alpha+\frac{\gamma}{1+\exp \{-\beta[\log (\mathrm{FDM})-\mu]\}}
$$

The lower asymptote $(\alpha)$ is a measure of the background level of absorbance corresponding with antigen-free sample. The upper asymptote $(\alpha+\gamma)$ is a measure of the saturation of the assay, and the difference between the lower and upper asymptote $(\gamma)$ determines the effective working range of absorbance values within which it is possible to detect differences in the amount of antigen present. Horizontal shifts in the calibration curve affect only the locational parameter $(\mu)$, while the rate parameter $(\beta)$ describes the steepness of the curve between the lower and upper asymptotes. A standard nonlinear fitting procedure (GENSTAT; Numerical Algorithms Group, Ltd., Oxford) was used to estimate the parameters.

Bias in an assay due to retention of the antigen is measured by the horizontal shift in the curve at the point of inflection and is estimated by the difference in the locational parameters $(\mu)$ for the test assay and the reference standard. The sensitivity of the assay is defined as the lower detection level (LDL) (milligrams per milliliter) of the antigen extracted from FDM that can just be distinguished by absorbance from the background signal. Bias, therefore, is a relative measure, whereas the LDL is absolute. The LDL is estimated by inverse prediction from the least significant difference between the absorbance of a test sample (assumed to be measured without error) and the background absorbance. The least signi- 
ficant difference is given by $\alpha+t s_{\alpha}$, in which $s_{\alpha}$ is the standard error of the lower asymptote and $t$ is the corresponding value for the $t$ distribution, yielding a LDL of

$$
\mathrm{LDL}=10^{k}
$$

$$
\text { in which } k=\mu+\frac{1}{\beta} \ln \left(\frac{t s_{\alpha}}{\gamma-t s_{\alpha}}\right)
$$

The goodness-of-fit of the model to the data was tested by approximate $F$ test for the ratio of lack-of-fit to pure error. Subject to a satisfactory fit (achieved in all cases), the standard error for $\alpha$ was based on the pure error or combined pure error and lack-offit, whichever was smaller.

PTA-ELISA. Wells of microtiter strips containing immobilized antigen were assayed by PTA-ELISA. Wells were blocked for 10 min with $100 \mu$ of Tris-buffered saline containing $0.3 \%$ (wt/vol) casein $(\mathrm{TBS} / \mathrm{c} ; 0.8 \% \mathrm{NaCl}, 0.02 \% \mathrm{KCl}, 0.3 \%$ Tris [T-1378; Sigma Chemical Co.], and $0.3 \%$ casein; $\mathrm{pH} 8.3$ ). Blocked wells were incubated sequentially, for $1 \mathrm{~h}$ each, with serum-free MAb supernatant from the cell line EE1 containing $0.3 \%$ (wt/vol) casein, followed by goat anti-mouse $\operatorname{IgM}$ ( $\mu$-chain specific) biotin conjugate (B-9265; Sigma Chemical Co.) diluted 1 in 3,000 in TBS/c. Wells were incubated for a further hour with Extravidin-peroxidase conjugate (E2886; Sigma Chemical Co.) diluted 1 in 1,000 in TBS/c, followed by substrate solution containing tetramethyl-benzidine for $30 \mathrm{~min}$. Color reactions were stopped by the addition of $3 \mathrm{M} \mathrm{H}_{2} \mathrm{SO}_{4}$ and absorbance values determined, at $450 \mathrm{~nm}$, using a MR700 micro-
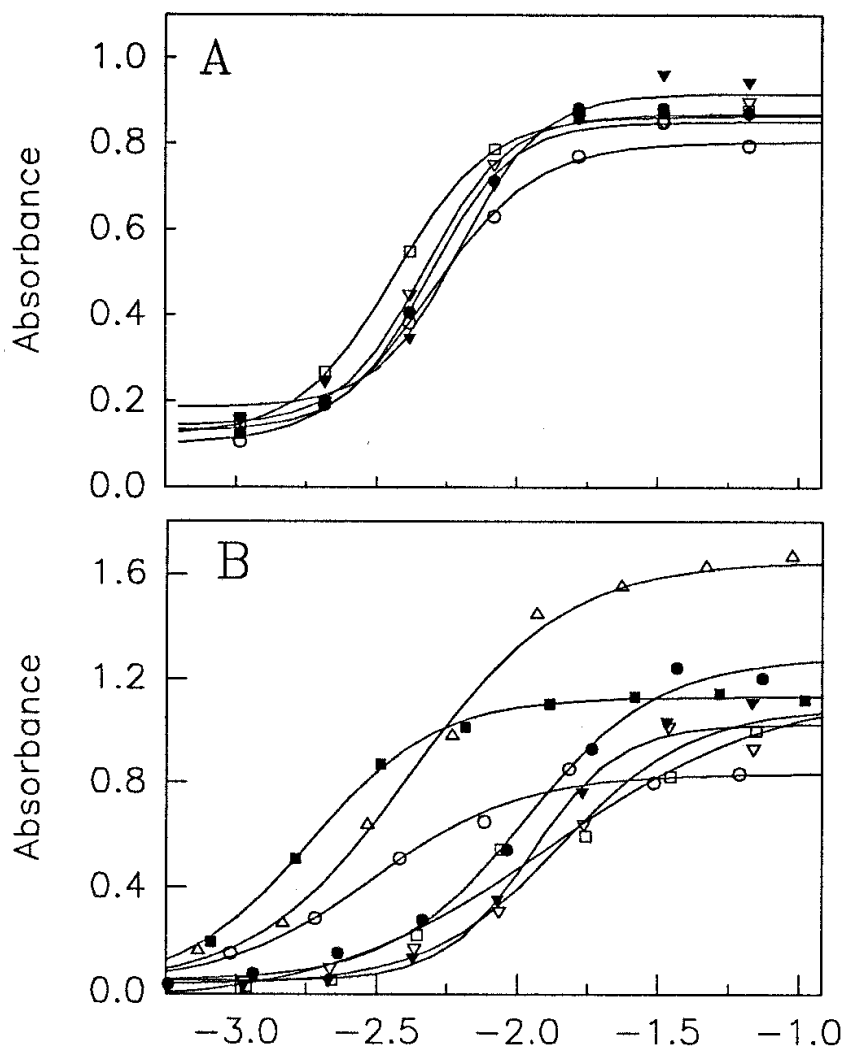

$\log [$ Freeze-dried mycelium] $(\mathrm{mg} / \mathrm{ml})$

Fig. 1. Relationship between serial dilutions of the antigen extracted from freeze-dried mycelium of Rhizoctonia solani and absorbance for A, identical series divided over five plates and analyzed on the same day; and $\mathbf{B}$, extracts prepared and analyzed on different days. Different symbols refer to mean absorbance values on A, separate plates or $\mathbf{B}$, separate days. Lines refer to fitted sigmoidal relationship according to equation 1 . plate autoreader (Bio-Tek Instruments, Inc., Winooski, VT) blanked against wells coated with buffer only. Working volumes were $50 \mu \mathrm{l}$ and wells were given four 3-min rinses with TBS/c between incubations and an additional 1-min rinse with distilled water prior to addition of substrate solution. All incubation steps were performed at $37^{\circ} \mathrm{C}$, with shaking (IncShake; Luminar Technology, Southampton, United Kingdom), in sealed plastic bags.

\section{RESULTS}

Reference calibration curve. Serial dilutions of the antigen from FDM dissolved in PBS alone resulted in an asymptotical sigmoidal relationship between the absorbance value and the loga-
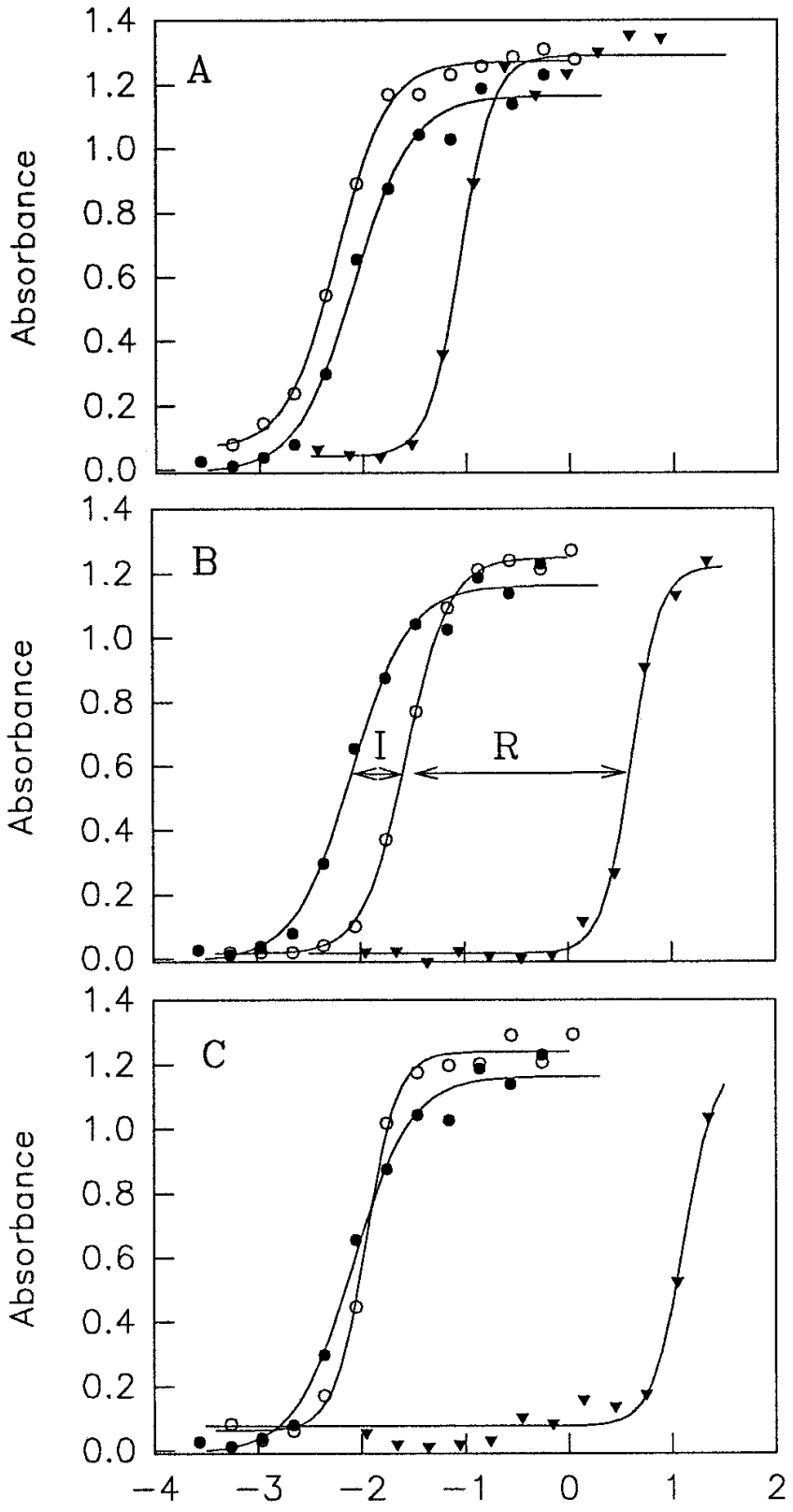

$\log [$ Freeze-dried mycelium] $(\mathrm{mg} / \mathrm{ml})$

Fig. 2. Mean data $(n=3)$ and fitted curves representing the relationship between the antigen extracted from freeze-dried mycelium of Rhizoctonia sola$n i$ and absorbance for the reference curve in phosphate-buffered saline (closed circles), in the presence of soluble components extracted from soil (open circles), and in the presence of soil (closed triangles) for A, sand; $\mathbf{B}$, loam; and $\mathbf{C}$, clay. The bias in the assay is defined as the horizontal displacement between calibration curves, and is identified as interference (I) or retention (R). 
rithm of the concentration of FDM (Fig. 1A). Calibration curves differed amongst plates assayed on the same day (Fig. 1A), with larger variation amongst plates assayed on different days (Fig. 1B). To minimize variability in the current study, treatments were timed so that all calibration curves were constructed and analyzed on the same day.

Bias due to interference. Absorbance values of antigen solutions serially diluted in soil extracts varied with soil type (Fig. 2, Table 1). For sand extract, absorbance values were slightly higher when compared with the standard curve in PBS (Fig. 2A), indicating positive interference of the soil components leading to a higher signal. The calibration curve for loam was markedly deflected to the right (Fig. 2B), indicating negative interference and a reduction in sensitivity of the assay. The curve for clay was not shifted horizontally, but rose more steeply ( $\beta$ greater for clay extract than for PBS, Fig. 2C). The LDLs varied correspondingly, ranging from $1.38 \times 10^{-3} \mathrm{mg} / \mathrm{ml}$ for sand to $4.68 \times 10^{-3} \mathrm{mg} / \mathrm{ml}$ for loam (Table 1).

Bias due to retention. The bias due to retention in the three test soils is shown in Figure 2 as the horizontal difference between the calibration curves prepared in soil extract and in bulk soil. The magnitude of the bias due to retention was substantially greater than the bias due to interference for all three soils. The curve for each soil was shifted to the right, indicating a reduction in sensitivity. The loss in sensitivity was greater in clay than in loam and least in sand (Fig. 2). Concentrations of the antigen extracted from FDM (milligrams per milliliter) that gave maximum absorbance values in PBS alone were not detectable after incorporation in the clay and the loam soil and could barely be detected in the sand (Fig. 2). An estimate of antigen recovery (Table 2) was calculated from the horizontal displacement parameters $(\mu)$, from which it may be seen that only a very small percentage $(<6.4 \%)$ of the antigen in soil was extracted with PBS.

The estimated values for the LDLs (Table 1) show that the combined effect of interference and incomplete extraction lead to at least a 1,000-fold reduction in the sensitivity of the assay (Table $2)$; the LDL for the reference sample in PBS was $1.78 \times 10^{-3}$ $\mathrm{mg} / \mathrm{ml}$, but rose to $1.48 \mathrm{mg} / \mathrm{ml}$ in the loam.

Improvement of the assay by increasing extraction. The sensitivity of the assay for the reference sample (without soil or soil extract) and the upper asymptote was reduced by the addition of $\mathrm{CuSO}_{4}$, indicating a negative effect on the assay. This negative effect, however, was compensated by a substantial reduction in the bias due to incomplete extraction, especially for the clay (Fig. 3). Extraction with $\mathrm{CuSO}_{4}$ increased recovery from all three soils
(Table 2). This markedly shifted the curves to the left, thereby improving the sensitivity of the soil assay, resulting in a reduced LDL (Table 1). The latter changed by more than 100-fold for clay, with a fourfold improvement for loam and almost twofold for sand (Table 2).

Reduction of interference by extract dilution. Increasing the ratio of buffer (either $\mathrm{CuSO}_{4}$ or PBS) to soil resulted in a rise and then a fall in absorbance for clay (Fig. 4B). This is consistent with a reduction in interference (leading to a rise in signal) that is offset by the reduction in antigen concentration. The corresponding curves for sand (Fig. 4A) did not exhibit an optimum, but the decrease in absorbance was less than would be expected if dilution of the antigen was the only mechanism operating (Fig. 2A, calibration curve). Extraction with $\mathrm{CuSO}_{4}$ in the sand reduced the absorbance values to lower levels. The improved extraction of the antigen from soil with $\mathrm{CuSO}_{4}$ (Table 2) was counteracted by a negative effect of $\mathrm{CuSO}_{4}$ on the assay (Fig. 3), resulting in lower absorbance values for this particular sample in sand. For extraction from the clay soil, however, the increase in extraction from soil was more important than the negative effect of $\mathrm{CuSO}_{4}$ on the assay, resulting in higher absorbance values. Note that although the absorbance values are low, the differences between the optimum and other points on the curve in Figure 4B are significant. The relative values for the optimum and other sections of the curve are the focus of attention here, not the absolute values. We conclude that the effect of interference can be reduced by increasing the ratio of buffer to soil and that there is an optimum ratio of buffer to clay soil of about 1.5 to $2.0 \mathrm{ml} / \mathrm{g}$.

\section{DISCUSSION}

We have shown that an antigenic extracellular catechol oxidase secreted by hyphae of $R$. solani is retained by soil components, giving rise to incomplete extraction from soil. This causes bias in the estimation of antigen concentration in soil samples and loss in sensitivity of a quantitative assay. When the assay is used for detection of the pathogen, this loss in sensitivity increases the risk of false negatives. Retention of the antigen in soil is substantially greater than bias due to interference of soil soluble components in the plate-trapped-antigen, enzyme-linked immunosorbent assay. The retention of the antigen in soil can reduce the sensitivity of the assay more than 1,000-fold for clay, and the degree of retention varies with different soils. It follows that separate calibration curves must be prepared for each soil in an assay. We have also shown that the sensitivity of an assay can be substantially in-

TABLE 1. The parameters for calibration curves fitted to a logistic function between absorbance and logarithm of the concentration of freeze-dried mycelium (milligrams per milliliter) and the lower detection level (LDL, milligrams of freeze-dried mycelium per milliliter) computed from these curves

\begin{tabular}{|c|c|c|c|c|c|c|c|c|}
\hline Antigen source ${ }^{v}$ & Extractant & Soil & $\beta^{w}$ & $\mu^{w}$ & $\gamma^{W}$ & $\alpha^{w}$ & LDL & Figure $^{\mathrm{x}}$ \\
\hline \multirow[t]{2}{*}{ Antigen in extractant (reference) } & PBS & $\ldots$ & $3.59(0.68)^{\mathrm{y}}$ & $-2.09(0.06)$ & $1.17(0.07)$ & $-0.006(0.05)$ & 0.00178 & $2,3 \mathrm{~A}$ \\
\hline & $\mathrm{CuSO}_{4}$ & $\ldots$ & $3.61(0.61)$ & $-1.47(0.05)$ & $0.80(0.05)$ & $0.019(0.02)$ & 0.00524 & $3 \mathrm{~B}$ \\
\hline \multirow[t]{6}{*}{ Antigen in soil extract } & PBS & Sand & $4.10(0.55)$ & $-2.25(0.04)$ & $1.21(0.05)$ & $0.068(0.05)$ & 0.00138 & $2 \mathrm{~A}$ \\
\hline & PBS & Loam & $4.79(0.35)$ & $-1.56(0.02)$ & $1.23(0.02)$ & $0.016(0.02)$ & 0.00468 & $2 \mathrm{~B}$ \\
\hline & PBS & Clay & $6.60(0.85)$ & $-1.96(0.02)$ & $1.18(0.03)$ & $0.063(0.03)$ & 0.00372 & $2 \mathrm{C}, 3 \mathrm{~A}$ \\
\hline & $\mathrm{CuSO}_{4}$ & Sand & $3.02(0.57)$ & $-1.66(0.07)$ & $1.05(0.07)$ & $0.037(0.05)$ & 0.00363 & $\ldots$ \\
\hline & $\mathrm{CuSO}_{4}$ & Loam & $4.10(0.65)$ & $-1.11(0.04)$ & $1.00(0.05)$ & $0.033(0.02)$ & 0.0138 & $\ldots$ \\
\hline & $\mathrm{CuSO}_{4}$ & Clay & $4.22(2.21)$ & $-1.62(0.14)$ & $0.67(0.10)$ & $0.056(0.06)$ & 0.0110 & $3 B$ \\
\hline \multirow[t]{6}{*}{ Antigen in soil } & PBS & Sand & $6.39(0.79)$ & $-1.06(0.02)$ & $1.25(0.03)$ & $0.043(0.03)$ & 0.0282 & $2 \mathrm{~A}$ \\
\hline & PBS & Loam & $7.15(0.93)$ & $0.62(0.02)$ & $1.20(0.04)$ & $0.018(0.02)$ & 1.48 & $2 \mathrm{~B}$ \\
\hline & PBS & Clay $^{z}$ & $6.61^{\mathrm{z}}$ & $1.11^{\mathrm{z}}$ & $1.14^{\mathrm{z}}$ & $0.076^{\mathrm{z}}$ & 3.98 & $2 \mathrm{C}, 3 \mathrm{~A}$ \\
\hline & $\mathrm{CuSO}_{4}$ & Sand & $2.92(0.61)$ & $-1.11(0.08)$ & $0.93(0.07)$ & $0.012(0.06)$ & 0.0162 & $\ldots$ \\
\hline & $\mathrm{CuSO}_{4}$ & Loam & $2.34(1.13)$ & $0.25(0.24)$ & $0.44(0.11)$ & $0.024(0.04)$ & 0.347 & $\ldots$ \\
\hline & $\mathrm{CuSO}_{4}$ & Clay & $2.46(0.59)$ & $-0.86(0.11)$ & $0.97(0.11)$ & $0.021(0.09)$ & 0.0355 & $3 B$ \\
\hline
\end{tabular}

\footnotetext{
${ }^{\mathrm{v}}$ The calibration curves are constructed in extractant (phosphate-buffered saline [PBS] or CuSO 4 ) only, in soil extract, or in soil.

${ }^{\mathrm{w}}$ The explanation of the parameters is in the text.

${ }^{x}$ Figure refers to the figures in which the calibration curves are shown.

y The standard errors of the parameters are given in parentheses.

${ }^{\mathrm{z}}$ Values not estimated accurately due to insufficient data on upper asymptote.
} 
creased with improved extraction of the antigen from soil, as in the current case by the addition of $\mathrm{CuSO}_{4}$. The results are based on a specific antigen-assay system, but have much broader implications for the design and use of calibration curves for assays involving soilborne fungal antigens.

In the absence of a source of purified antigen, extracts derived from FDM were used as a quantifiable and repeatable source of antigen to compare different extraction techniques. Large quantities of mycelium can be conveniently prepared and stored as a bulk source, remaining stable at low temperature for many years (5). Much still needs to be done to relate the ecological activity of mycelium in soil with the absorbance obtained from FDM, but this is beyond the scope of the current study. Strict control and repeatability of analyses is an essential first step in devising and testing protocols for the efficient use of MAb-based assays. Without this preliminary step, important mechanisms that affect the bias and sensitivity of assays may be obscured, and the opportunity to ameliorate the effects of bias may consequently be missed.

The relationship between antigen and absorbance is highly nonlinear; successive increments of antigen elicit markedly different responses in absorbance. The sigmoidal-shaped relationship, familiar in assays of this form $(4,10,13)$, was adequately described by the logistic function. Day-to-day variation and variation among plates in ELISA-based systems is common $(3,7,21)$, but mechanisms that cause this phenomenon are still unknown. Daily variation changed the shape of the calibration curves (Fig. 1), instead of a simple vertical or horizontal displacement of the curves. It is, therefore, essential that calibration curves include the full range of antigen concentration between the upper and lower limits for absorbance. Reliance on a single sample of known antigen content for calibration is not sufficient (21). Despite this, we note that the use of calibration curves and the conversion of absorbance values to amounts of antigen are surprisingly absent from many studies involving enzyme immunoassays. We advocate a simple protocol for the assay of antigens in soil-based systems that involves preparation of calibration curves in antigen-free soil that is otherwise identical to the test soil, with separate curves for each assay time and ideally for each plate. An antigen-free sample may be easily obtained for laboratory studies in controlled experiments, but how such a sample can be obtained for analysis of a field sample requires further investigation.

We identified two principal criteria in the calibration of an immunoassay. One is the LDL, computed as the least significant difference from the computed lower asymptote. The absorbance of the test sample is here assumed to be measured without error, for ease of comparison amongst different treatments. Variability in the test sample is easily incorporated into the least significant difference. The LDL is an absolute measure of the threshold concentration that can be distinguished from the background. The second criterion involves bias, which is a relative measure, because it is compared with an arbitrarily selected standard. We use

TABLE 2. The mean percentage of antigen recovered from three soils, the loss in sensitivity compared with a calibration in the absence of soil, and the improved sensitivity for extraction with $\mathrm{CuSO}_{4}$ compared with extraction with phosphate-buffered saline (PBS)

\begin{tabular}{lccccc}
\hline & \multicolumn{2}{c}{ PBS } & & \multicolumn{2}{c}{$\mathrm{CuSO}_{4}$} \\
\cline { 2 - 3 } \cline { 5 - 6 } Soil type & $\begin{array}{c}\text { Recovery } \\
(\%)^{\mathrm{y}}\end{array}$ & $\begin{array}{c}\text { Loss in } \\
\text { sensitivity }\end{array}$ & & $\begin{array}{c}\text { Recovery } \\
(\%)\end{array}$ & $\begin{array}{c}\text { Improved } \\
\text { sensitivity }\end{array}$ \\
\hline Sand & 6.4 & $\times 16$ & & 28.2 & $\times 1.7$ \\
Loam & 0.7 & $\times 830$ & & 4.4 & $\times 4.3$ \\
Clay & $<0.1$ & $\times 2,236$ & & 17.4 & $\times 112$ \\
\hline
\end{tabular}

${ }^{y}$ The recovery is calculated from the horizontal displacement between the calibration curves in soil extract and in soil at the point of inflection $(\mu$ parameter).

${ }^{z}$ The relative measures of sensitivity are calculated from the difference in the lower detection levels. the locational parameter, $\mu$, of the logistic function (8) to characterize the midpoint of the range of the antigen over which there are detectable changes in absorbance. The magnitude of bias of a test curve (e.g., for clay extracted in $\mathrm{CuSO}_{4}$ ) is then measured relative to a reference standard as the difference between the locational parameters.

We identified two sources that lead to bias in the quantification of antigens in soil: (i) interference of soluble components and (ii) retention of the antigen. In practice, the amount of bias in the PTA-ELISA due to interference of soluble components was small relative to the bias and loss of sensitivity due to retention in soil (Figs. 2 and 3). The interference resulting from soluble components may appear positive (curve shifted to the left) or negative (curve shifted to the right), because it is compared with an arbitrarily selected standard, in this case PBS. Organic acids extracted from soil may compete with the antigen for binding to the microtiter well. Increasing the $\mathrm{pH}$, which increases extraction of organic matter from soils (17), significantly reduced the sensitivity of the assay in the clay in our studies (W. Otten, unpublished data). Attempts to remove humic contaminants from the soil solution with polyvinylpolypyrrolidone (11) and by heating the solution, however, had deleterious effects on assay sensitivity. The loss in sensitivity with heating was most likely due to denaturation of the antigen. Some authors advocate the use of a double-antibody sandwich (DAS)-ELISA to minimize interference due to soluble components (12). We argue that this may reduce interference, but has no effect on extraction of the antigen from soil, which we have identified here as the major factor limiting the sensitivity of the assay. In our particular case, the use of a DASELISA failed because this assay format was less sensitive, in par-
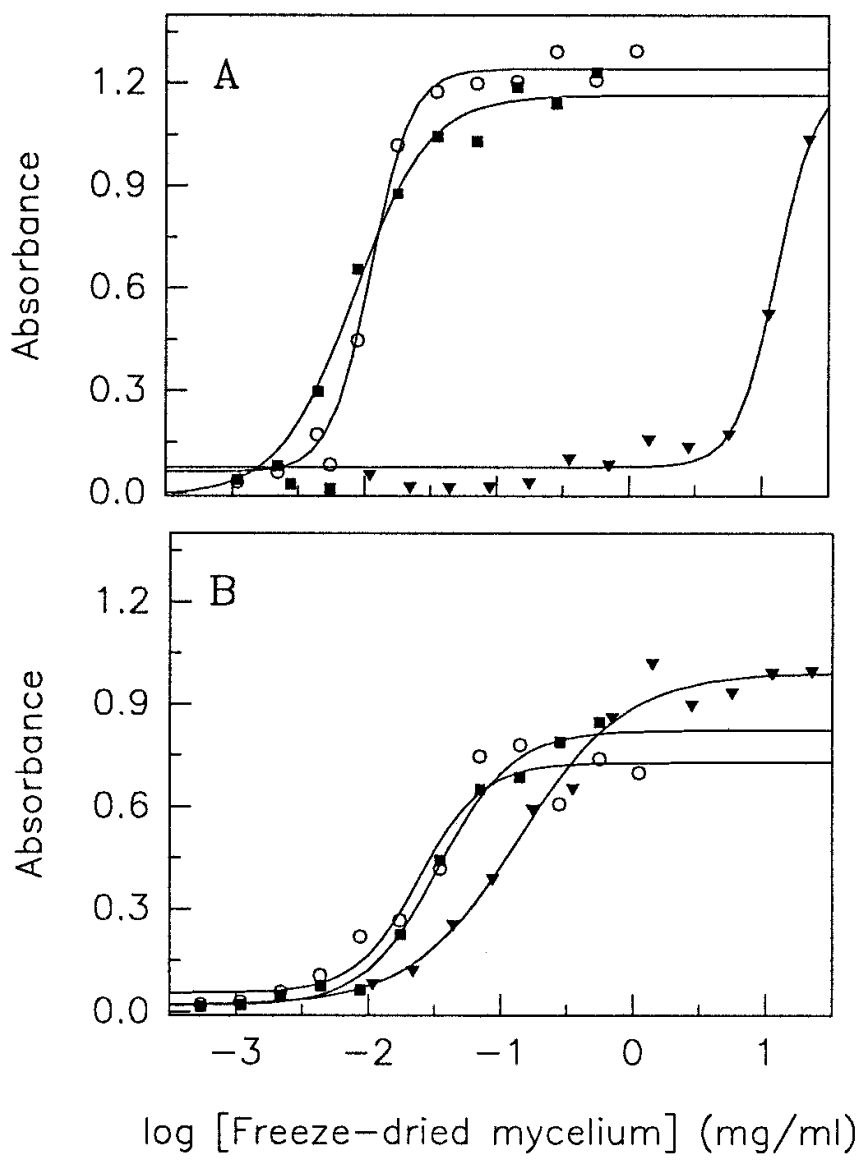

Fig. 3. Mean data $(n=3)$ and fitted calibration curves using $\mathbf{A}$, phosphatebuffered saline and $\mathbf{B}, \mathrm{CuSO}_{4}$ for detection of the antigen extracted from freeze-dried mycelium of Rhizoctonia solani dissolved in extractant (closed squares), in clay extract (open circles), or in clay (triangles). 
ticular when $\mathrm{CuSO}_{4}$ was used to increase extraction from soils (W. Otten, unpublished data).

Retention of the antigen in soil accounted for most of the bias in estimating antigen concentration in all three soils (Fig. 2, horizontal displacement of the curves). The mechanisms responsible for retention of the antigen in soil are not yet well understood. Attempts to block adsorption sites in the soils were largely unsuccessful. Adding divalent salts, detergents, excess of phosphate, paraquat (1,1'-dimethyl-4,4'-bipyridylium dichloride), sulfate, EDTA, or altering the $\mathrm{pH}$ or ionic environment failed to increase sensitivity (W. Otten, unpublished data). While these extractants may have increased the amounts of antigen extracted, they are likely also to have caused structural changes to the glycoprotein molecule itself, thereby inhibiting binding of the antibody to its epitope.

Characterization of the source of bias in immunoassays indicates how sensitivity can be improved. Addition of $\mathrm{CuSO}_{4}$ to the extraction solution reduced the bias and increased the sensitivity of the assay in all three soils (Table 1, Fig. 3). Similar results were obtained with zinc (W. Otten, unpublished data). Both $\mathrm{Cu}^{2+}$ and $\mathrm{Zn}^{2+}$ form soluble and insoluble complexes with organic matter (2). This may affect potential binding of the antigen onto organic matter in soil, or inhibit organic matter from binding to the microtiter well and, therefore, reduce interference. In addition, $\mathrm{Cu}^{2+}$ and $\mathrm{Zn}^{2+}$ adsorb preferentially onto clay minerals (2) and to fungal biomass, with $\mathrm{Cu}^{2+}$ adsorbed more favorably than $\mathrm{Zn}^{2+}$ (14). Blocking of potential binding sites is, therefore, a plausible explanation for the successful use of $\mathrm{Cu}^{2+}$ in extracting antigens from clay soil, which had the highest clay and organic matter content of the soils used in this study. Further investigation into the exact nature of
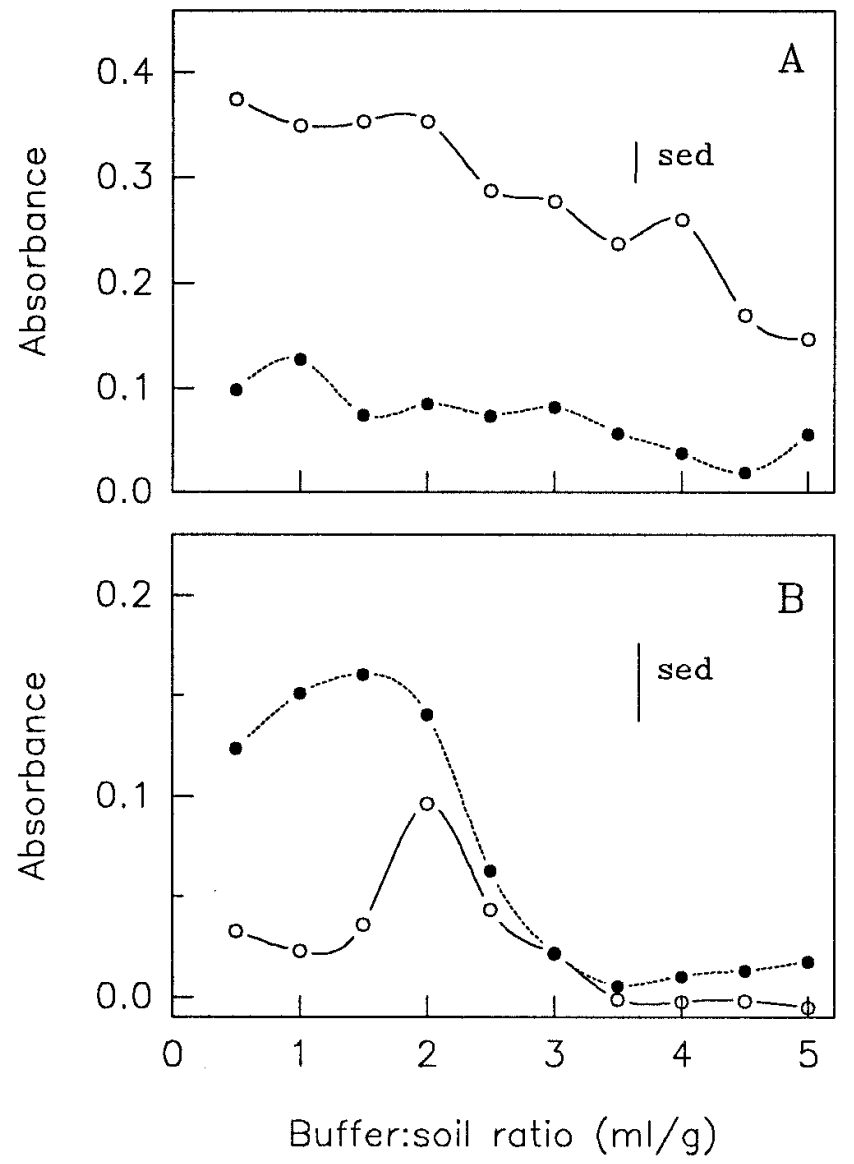

Fig. 4. Mean absorbance measured for $\mathbf{A}$, sand or $\mathbf{B}$, clay-sand mixture infested with Rhizoctonia solani in relation to the extraction volume (milliliter of extractant per gram of soil). The samples were extracted with phosphatebuffered saline (open circles) or $\mathrm{CuSO}_{4}$ (closed circles). The standard errors of difference (sed) are given for individual means. The curves were obtained by spline-fitting. the binding may help to find procedures to increase extraction. The relative success of $\mathrm{Cu}^{2+}$ in this assay may also be associated with the nature of the antigen used in this study, a putative catechol oxidase, which contains copper as a functional group (16). Gouzou (9) found that addition of aluminum or strontium to extraction buffers increased the sensitivity of an antigen-coated plate immunoassay for the enumeration of cells of Bacillus polymyxa due to an increased extraction from soil.

The sensitivity of the assay was further improved by optimizing the volume of extractant. With increasing volume of extractant, the concentration of components that can interfere in the assay is reduced, and so the bias in the quantification is also reduced. Concomitantly, the antigen concentration is diluted, thereby decreasing the absorbance value. Here, the critical test is whether or not there is an optimum for the absorbance dilution ratio. We found that the optimal extraction procedure depended on soil type, and propose a low extraction ratio with PBS for sand, and a slightly larger extraction ratio with $\mathrm{CuSO}_{4}$ solution for clay soil (Fig. 4).

The work described in this paper demonstrates that quantification of fungal antigen can be successfully achieved in soil using MAbs. The sensitivity of a potentially good assay is substantially decreased when applied to soil. Calibration curves described in this paper give quantitative insight into sources of loss of sensitivity for soil-based assays and, therefore, form the basis for assay improvement. Retention of antigens in soil was identified as a major source for loss in sensitivity. Bias due to retention of antigens in soil and interference of soluble components has been reduced by extracting with $\mathrm{CuSO}_{4}$ and by optimizing the volume of the extraction solution. Further improvements in assay sensitivity are possible. We advocate the need for a better understanding of the molecular structure of fungal antigens and the nature of their binding to soil particles. This will facilitate the development of methods for improved recovery of antigens from soil. Further, it will enable more efficient screening, during initial tests of hybridoma cell lines, to select antibodies that recognize antigens that can be readily extracted from soil. Overall, improvement of the sensitivity of an assay can best be achieved by optimizing the entire system, including the raising of antibodies, the extraction of antigens from soil, and the format of the enzyme immunoassay employed.

\section{ACKNOWLEDGMENTS}

This work was funded by grants from the Biotechnology and Biological Sciences Research Council (BBSRC), which we acknowledge. The monoclonal antibodies were raised in a collaborative project, funded by the BBSRC, with F. M. Dewey, Department of Plant Sciences, University of Oxford, which we also acknowledge.

\section{LITERATURE CITED}

1. Avila, F. J., Yuen, G. Y., and Klopfenstein, N. B. 1995. Characterization of a Pythium ultimum-specific antigen and factors that affect its detection using a monoclonal antibody. Phytopathology 85:1378-1387.

2. Baker, D. E., and Amacher, M. C. 1982. Nickel, copper, zinc, and cadmium. Pages 323-336 in: Methods of Soil Analysis: Part 2 Chemical and Microbiological Properties. 2nd ed. Agronomy Series No. 9. A. L. Page, ed. American Society of Agronomy, Inc., Madison, WI.

3. Bauske, E. M., Hewings, A. D., Kolb, F. L., and Carmer, S. G. 1994. Variability in enzyme-linked immunosorbent assays and control of experimental error by use of experimental designs. Plant Dis. 78:1206-1210.

4. Bossi, R., Cole, L., Spier, A. D., and Dewey, F. M. 1994. Monoclonal antibody-based ELISA for detection of mycelial antigens of Botrytis cinera in fruits and vegetables. Pages 165-172 in: Modern Assays for Plant Pathogenic Fungi: Identification, Detection and Quantification. A. Schots, F. M. Dewey, and R. Oliver, eds. CAB International, Wallingford, United Kingdom.

5. Dewey, F. M., and Thornton, C. R. 1995. Fungal immunodiagnostics in plant agriculture. Pages 151-171 in: New Diagnostics in Crop Sciences. Biotechnology in Agriculture No. 13. J. H. Skerritt and R. Appels, eds. CAB International, Wallingford, United Kingdom.

6. Dewey, F. M., Thornton, C. R., and Gilligan, C. A. 1996. Use of monoclonal antibodies to detect, quantify and visualize fungi in soils. Adv. Bot. Res. 24:275-308. 
7. Dewey, F. M., Twiddy, D. R., Phillips, S. I., Grose, M. J., and Wareing, P. W. 1992. Development of a quantitative monoclonal antibody-based immunoassay for Humicola ianuginosa on rice grains and comparison with conventional assays. Food Agric. Immunol. 4:153-167.

8. Gilligan, C. A. 1990. Comparison of disease progress curves. New Phytol. 115:223-242.

9. Gouzou, L. 1992. Devenir d'une population bactérienne inoculée dans la rhizosphère du blé et ses effects sur la plante: Cas de Bacillus polymyxa. (The dynamics of Bacillus polymyxa in the wheat rhizosphere after inoculation and its effects on the plant growth, English abstract) Ph.D. thesis. Université de Nancy I, Nancy, France.

10. Harrison, J. G., Lowe, R., Wallace, A., and Williams, N. A. 1994. Detection of Spongospora subterranea by ELISA using monoclonal antibodies. Pages 23-28 in: Modern Assays for Plant Pathogenic Fungi: Identification, Detection and Quantification. A. Schots, F. M. Dewey, and R. Oliver, eds. CAB International, Wallingford, United Kingdom.

11. Holben, W. E. 1994. Isolation and purification of bacterial DNA from soil. Pages 727-752 in: Methods of Soil Analysis, Part 2: Microbiological and Biochemical Properties. Book Series No. 5. R. W. Weaver, J. S. Angle, and P. S. Bottomley, eds. Soil Science Society of America, Inc., Madison, WI.

12. Holtz, B. A., Karu, A. E., and Weinhold, A. R. 1994. Enzyme-linked immunosorbent assay for detection of Thielaviopsis basicola. Phytopathology 84:977-983.

13. Miller, S. A., Rittenburg, J. H., Petersen, F. P., and Grothaus, G. D. 1992. From the research bench to the market place: Development of commercial diagnostic kits. Pages 208-221 in: Techniques for the Rapid Detection of Plant Pathogens. J. M. Duncan and L. Torrance, eds. Blackwell Scientific Publications Ltd., Oxford.

14. Morley, G. F., and Gadd, G. M. 1995. Sorption of toxic metals by fungi and clay minerals. Mycol. Res. 99:1429-1438.

15. O'Brien, P. A. 1996. Identification and detection of Rhizoctonia solani using serological and DNA marker techniques. Pages 177-185 in: Rhizoctonia Species: Taxonomy, Molecular Biology, Ecology, Pathology and Disease Control. B. Sneh, S. Jabaij-Hare, S. Neate, and G. Dijst. eds. Kluwer Academic Publishers, Dordrecht, the Netherlands.

16. Sató, M. 1980. Reactivation by copper of phenolase pre-inactivated by oxalate. Phytochemistry 19:1931-1933.

17. Schnitzer, M. 1982. Organic matter characterization. Pages 581-594 in: Methods of Soil Analysis: Part 2 Chemical and Microbiological Properties. 2nd ed. Agronomy Series No. 9. A. L. Page, ed. American Society of Agronomy, Inc., Madison, WI.

18. Thornton, C. R., Dewey, F. M., and Gilligan, C. A. 1993. Development of monoclonal antibody-based immunological assays for the detection of live propagules of Rhizoctonia solani in soil. Plant Pathol. 42:763-773.

19. Thornton, C. R., Dewey, F. M., and Gilligan, C. A. 1994. Development of monoclonal antibody-based immunological assays for the detection of live propagules of Rhizoctonia solani in soil. Pages 29-36 in: Modern Assays for Plant Pathogenic Fungi: Identification, Detection and Quantification. A. Schots, F. M. Dewey, and R. Oliver, eds. CAB International, Wallingford, United Kingdom.

20. Wallace, A., Williams, N. A., Lowe, R., and Harrison, J. G. 1995. Detection of Spongospora subterranea using monoclonal antibodies in ELISA. Plant Pathol. 44:355-365.

21. Wright, S. F. 1994. Serology and conjugation of antibodies. Pages 593618 in: Methods of Soil Analysis, Part 2: Microbiological and Biochemical Properties. Book Series No. 5. R. W. Weaver, J. S. Angle, and P. S. Bottomley, eds. Soil Science Society of America, Inc., Madison, WI.

22. Yuen, G. Y., Craig, M. L., and Avila, F. 1993. Detection of Pythium ultimum with a species-specific monoclonal antibody. Plant Dis. 77:692-698. 